\title{
Efficacy of Phosphorus Gettering and Hydrogenation in Multicrystalline Silicon
}

\author{
Sarah Gindner, Philipp Karzel, Bernhard Herzog, and Giso Hahn
}

\begin{abstract}
The emitter formation step ( $\mathrm{POCl}_{3}$ diffusion) in p-type crystalline silicon solar cell processing includes many variables, e.g., peak temperature, gas flows, temperature ramps, which can be optimized in order to improve material quality. Diffusion parameters of an $80-\Omega / \square$ emitter are varied, and the resulting change in electronic quality of multicrystalline silicon is analyzed. A detailed gettering analysis of multicrystalline material, surface passivated with hydrogen-rich amorphous silicon, after $\mathrm{POCl}_{3}$ diffusion, and an additional gettering step combined with hydrogenation from $\mathrm{SiN}_{x}: \mathrm{H}$ is presented. The industrial-type diffusion leads to material of lower electronic quality than the extended reference diffusion. A major finding of this paper is the fact that results on different $5 \times 5 \mathrm{~cm}^{2}$ samples out of one $15.6 \times 15.6 \mathrm{~cm}^{2}$ wafer can vary significantly. Hence, conclusions about which diffusion is most efficient in gettering strongly depend on wafer position. An edge position close to crucible walls, for example, might improve less effectively than another position close to the crucible center. In fact, the opposite can also be shown, and samples originating from edge regions reach their highest lifetimes after gettering. This is explained by the different defect structure of the investigated samples. Structures exhibiting high gettering efficacy contain fewer recombination active grain boundaries and are predominantly free of extended defect clusters.
\end{abstract}

Index Terms-Gettering, hydrogen, photoconductivity, photoluminescence (PL), photovoltaic cells, silicon.

\section{INTRODUCTION}

$\mathbf{M}$ ULTICRYSTALLINE silicon (mc-Si) is of high industrial relevance because of its lower production costs compared with single crystalline silicon growth methods like float-zone and Czochralski. Because of the specific cast process, a high amount of defects, e.g., impurities, vacancies, grain boundaries (GBs), and dislocations are present in the material. A tremendous improvement of material quality is reported during the emitter formation step of p-type silicon solar cells using a $\mathrm{POCl}_{3}$ diffusion [1]-[3]. This effect is commonly known as phosphorus diffusion gettering (PDG). The detailed mechanisms behind this effect are still not clear up to now and still highly discussed. This is not surprising due to the complexity of the process. Each multicrystalline material contains a large variety of impurity species [4], [5], e.g., $\mathrm{Fe}, \mathrm{Ni}, \mathrm{Cu}, \mathrm{Co}, \mathrm{Cr}$, etc., with different diffusion properties and solubility in silicon

The authors are with the Department of Physics, University of Konstanz, 78464 Konstanz, Germany (e-mail: sarah.gindner@uni-konstanz.de; philipp.karzel@uni-konstanz.de; bernhard.herzog@uni-konstanz.de; giso. hahn@uni-konstanz.de). and exhibits different grain boundary structures decelerating or accelerating impurity diffusion [6]. In addition to the already present defects in the material, the applied $\mathrm{POCl}_{3}$ diffusion delivers oxygen and phosphorus which diffuse into the silicon matrix [7]-[9]. There are not only single impurity atoms in the silicon, but also complexes of two or more atoms are formed and precipitation occurs at certain fixed nucleation sites (preferably at dislocations, GBs, and extended defects [10], [11]). A measure of electronic material quality is the minority carrier lifetime determined by, e.g., quasi-steady-state photoconductance (QSSPC) [12] and spatially resolved photoluminescence (PL) [13] measurements. A lot of scientific effort was made to determine most lifetime limiting defects and to learn to remove them most effectively [14]-[17].

In this study, these techniques are used to analyze the gettering efficacy of two $80-\Omega / \square$ emitters with different process durations and gas flows. To achieve this, it is essential to understand the mechanisms behind PDG [18]-[21]. Multicrystalline samples of the same cast ingot differing significantly by their grain structure are selected. Therefore, a detailed gettering analysis on these samples is carried out, and the correlation between defect structure and gettering efficacy is investigated.

\section{EXPERIMENT}

In this investigation, four different process sequences are applied to four vertically directly neighboring p-type mc-Si samples $(\approx 1 \Omega \mathrm{cm}, \approx 150 \mu \mathrm{m})$ with comparable grain structure and very similar defect distribution (in the following referred to as sister samples):

1) Diffusion 1: reference;

2) Diffusion 2: industry;

3) Diffusion $1+H$ : reference gettering and subsequently firing of emitter and $\mathrm{SiN}_{x}: \mathrm{H}$ layer;

4) Diffusion $2+H$ : industry gettering and subsequently firing of emitter and $\mathrm{SiN}_{x}: \mathrm{H}$ layer.

If $\mathrm{SiN}_{x}: \mathrm{H}$ is additionally deposited on both sides in an industrial-type plasma-enhanced chemical vapor deposition (PECVD) system and fired, processes are denoted as diffusion + $H$. Therefore, in Fig. 1(b), all samples are schematically divided into groups A (without $\mathrm{SiN}_{x}: \mathrm{H}$ ) and $\mathrm{B}$ (with $\mathrm{SiN}_{x}: \mathrm{H}$ ). Note that group B samples are fired with their emitter layers still present. Thus, both effects during deposition and firing, i.e., the additional gettering as well as the hydrogenation, cannot be separated. On the other hand, this allows a direct comparison with solar cell material that is presented in Section III-D. as the final solar cell performance is affected by several processing steps (gettering and hydrogenation). In central ingot height, two sister samples per process are investigated to improve statistics. 
(a)

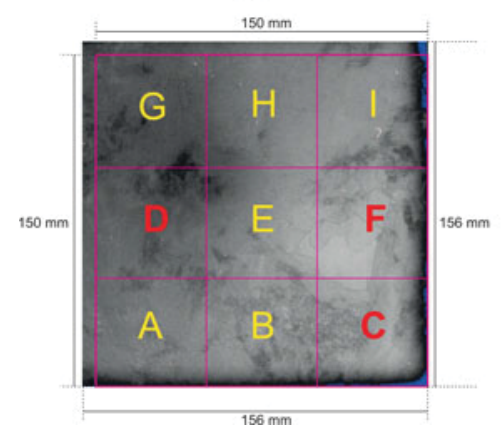

(b)

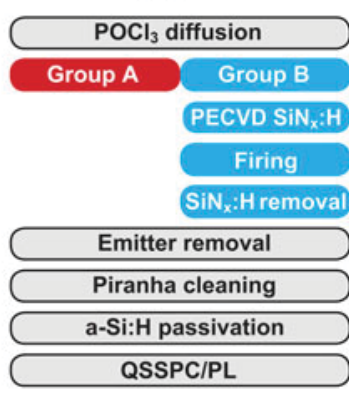

Fig. 1. (a) PL image of a $15.6 \times 15.6 \mathrm{~cm}^{2} \mathrm{mc}-\mathrm{Si}$ wafer out of a Gen 1 crucible (as-cut). (b) Process flow of lifetime samples. (a) Cutting sketch. (b) Process flow.

Samples are cleaned by a polishing etch consisting of HF, $\mathrm{HNO}_{3}$, and $\mathrm{CH}_{3} \mathrm{COOH}$. According to the process flow in Fig. 1(b), two 80- $\Omega / \square \mathrm{POCl}_{3}$ emitters with different process durations and gas flows are applied. More information on the diffusion parameters is given in Section III-A. After emitter removal (additionally for group B removal of $\mathrm{SiN}_{x}: \mathrm{H}$ layers) and surface piranha cleaning containing $\mathrm{H}_{2} \mathrm{O}_{2}$ and $\mathrm{H}_{2} \mathrm{SO}_{4}$ followed by HF dip, samples are surface passivated. The applied surface passivation is hydrogen-rich amorphous silicon (a-Si:H) [22] deposited in a PECVD system from Oxford Instruments (Plasmalab 100). Finally, lifetime characterization is performed using the QSSPC and PL techniques. PL images are measured at a photon generation flux of $2.6 \times 10^{17} \mathrm{~cm}^{-2} \mathrm{~s}^{-1}$. These images are lifetime-calibrated using the QSS lifetime.

The analysis is executed on $5 \times 5 \mathrm{~cm}^{2}$ samples originating from a mc-Si ingot of Gen 1 size produced within the framework of the project SolarWinS (see acknowledgment). Gen 1 size means that one column with an area cross section of $15.6 \times 15.6 \mathrm{~cm}^{2}$ is obtained from one crystallization process. This column is sawed into wafers, and each of them is subsequently cut into nine samples $\left(5 \times 5 \mathrm{~cm}^{2}\right)$, positioned as shown in Fig. 1(a). The analyzed positions are marked in red: C, D, and F. The shown PL image of the wafer reveals that the edges to the right $(\mathrm{C}, \mathrm{F}, \mathrm{I})$ and to the bottom are close to crucible walls with impurities diffusing in from these walls, reducing the wafer quality during crystal growth. This region of lower material quality is often called red zone. The already described wafer positions are from three different ingot heights: bottom, center, and top.

\section{POCL $\mathrm{P}_{3}$ GetTering ANALysis}

\section{A. Applied POCl 3 Diffusions: Reference and Industry}

In Fig. 2, two varied $\mathrm{POCl}_{3}$ diffusions applied in this study are sketched. Both processes lead to a sheet resistance of $80 \Omega / \square$. The red continuous line marks the reference diffusion. This is the standard emitter of laboratory-sized solar cells, whose front contact grid is defined by photolithography. In comparison with the industry diffusion drive-in of $5 \mathrm{~min}$, the reference drivein lasts considerably longer with $60 \mathrm{~min}$, and its oxygen flow

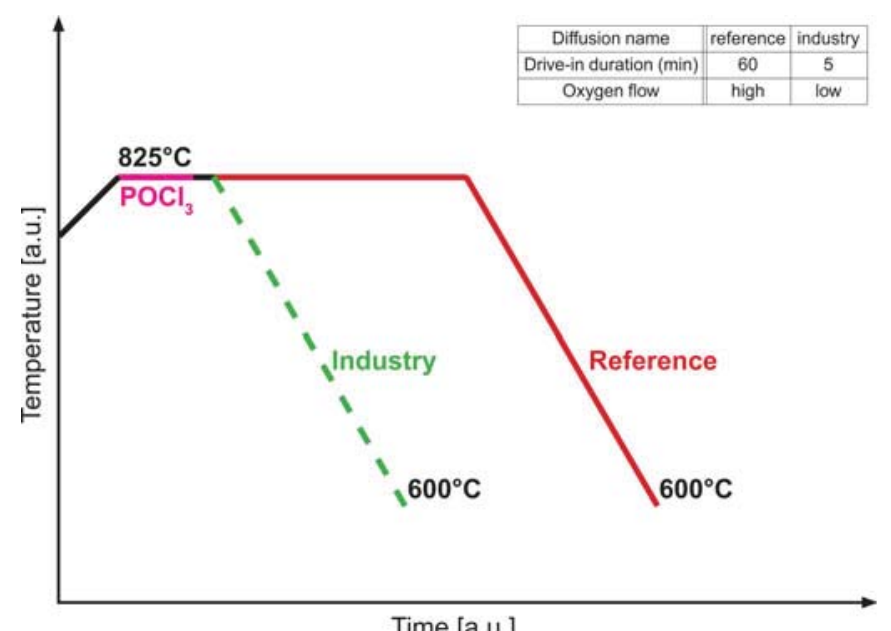

Time [a.u.]

Fig. 2. Applied $\mathrm{POCl}_{3}$ diffusions.

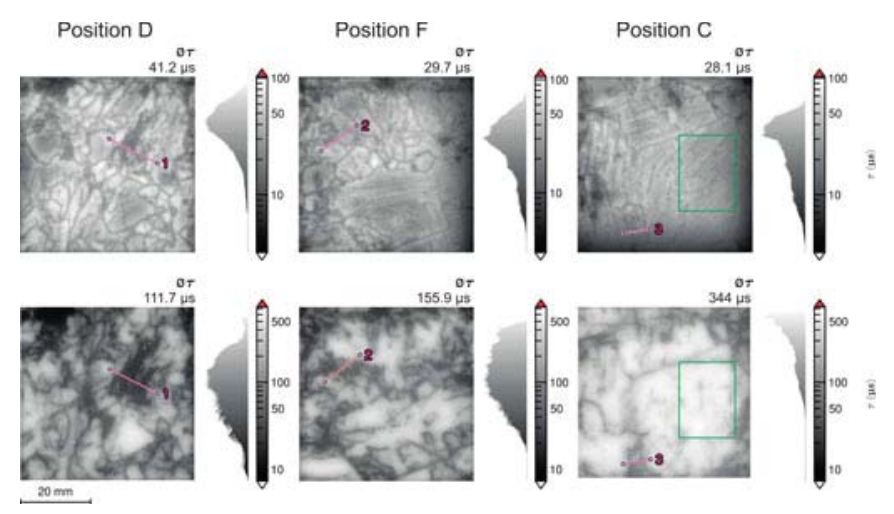

Fig. 3. PL maps of different positions before (as-grown, top row) and after industry diffusion (bottom row). On each sample, linescans of Fig. 5 are marked as 1 to 3 . On position $\mathrm{C}$, an image section is highlighted in green and depicted in Fig. 4. In addition, the arithmetically averaged lifetime is given.

is higher (see the table in Fig. 2). Unloading temperature is $600{ }^{\circ} \mathrm{C}$ for both processes.

\section{B. Detailed Gettering Analysis in Central Ingot Height Using Linescans}

This section focuses on gettering efficacy of three different positions C, D, and F (see Fig. 3). According to Fig. 1(a), positions $\mathrm{C}$ and $\mathrm{F}$ originate from ingot edge regions, while position $\mathrm{D}$ is closer to the ingot center. This is in agreement with the lower as-grown mean lifetimes $(\approx 29 \mu \mathrm{s})$ of these positions compared with $41 \mu$ s of sample D. A dark PL contrast at the right and bottom edges is due to the proximity to crucible walls serving as a source of contamination. At first sight, as-grown samples exhibit different defect structures. From left to right in the top row of Fig. 3, grain sizes increase, and therefore, the amount of GBs decreases. Whereas defect clusters [23], which are localized dark regions of random shape, are mainly observed for positions $\mathrm{D}$ and $\mathrm{F}$, position $\mathrm{C}$ is almost free of such clusters. After industry diffusion gettering, a stronger PL contrast is predominantly visible at these clusters (note the different scaling). 


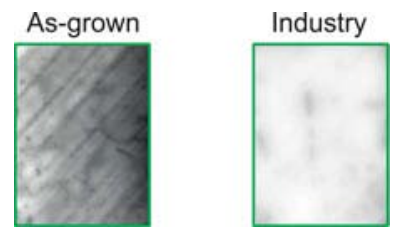

Fig. 4. Green rectangles in Fig. 3. Enlarged image details of position C before and after industry diffusion. GBs running parallel mainly observed for position $\mathrm{C}$ in the as-grown state.

Hence, the highest mean lifetime of $344 \mu$ s after gettering and, by far, the highest gettering efficacy is detected on position $\mathrm{C}$. This suggests that defect clusters hinder the gettering process. It is interesting to note that GBs of weaker PL contrasts in the as-grown images, running parallel, vanish after gettering. To examine this in more detail, an image section of position $\mathrm{C}$ is marked as green rectangle in Fig. 3 and depicted in Fig. 4. Because of their specific structure, these GBs might accommodate impurities with low activation energies so that gettering is facilitated. This is discussed in more detail in Section IV. Another explanation for this observation could be the reduction and/or removal of structural defects due to the high-temperature diffusion step or longer diffusion durations. Fenning et al. [24] reported a reduction of the dislocation density with higher diffusion temperature already in the range of $820-920{ }^{\circ} \mathrm{C}$.

In addition to the already gained insight into gettering mechanisms, one linescan is performed on each wafer position, and marked as 1 to 3 in Fig. 3. The scans across selected defect clusters are depicted in Fig. 5. The already stated higher lifetime contrast after industry diffusion compared with the as-grown state is also confirmed by these linescans. A useful parameter, in order to quantify clusters appearing in PL images by their recombination activity, is the $\mathrm{PL}$ contrast $C$. This quantity is calculated from the lifetime plateaus to the left $\tau_{\text {left }}$ and right $\tau_{\text {right }}$ of a linescan profile and from its minimum lifetime $\tau_{\text {min }}$ [see Fig. 5(a)]

$$
C=\frac{\bar{\tau}_{\text {margins }}-\tau_{\text {min }}}{\bar{\tau}_{\text {margins }}}
$$

with

$$
\bar{\tau}_{\text {margins }}=\frac{\tau_{\text {left }}+\tau_{\text {right }}}{2} .
$$

All positions $\mathrm{D}, \mathrm{F}$, and $\mathrm{C}$ exhibit a significantly lower lifetime within the scanned clusters $\tau_{\min }$ after industry diffusion than after reference diffusion. Besides that, profiles after both diffusions are similarly shaped and are even not distinguishable toward the cluster margins. This results in a higher lifetime contrast for the industry diffusion. From the blue-dotted line representing the industry diffusion, a contrast value $C$ is calculated, e.g., $90 \%$ for position D. The lifetime contrast of the reference diffusion depicted as red dotted line is $65 \%$, which is considerably lower. Consequently, the longer lasting reference diffusion removes impurities more effectively out of defect clusters.

The as-grown linescan only slightly resembles the profiles after gettering. This might be due to the as-grown material containing a high amount of homogeneously distributed impurities.

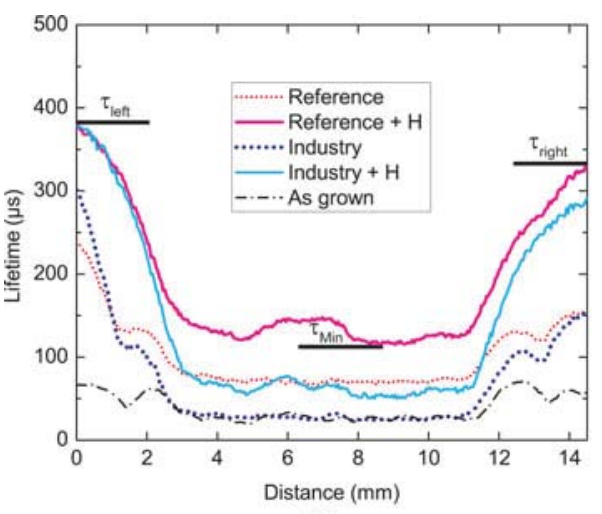

(a)

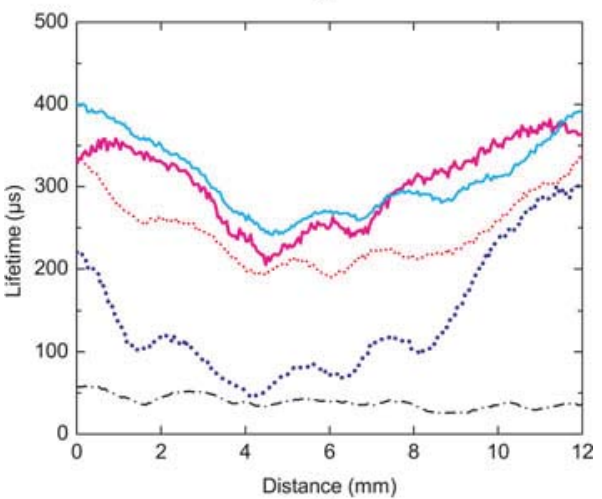

(b)

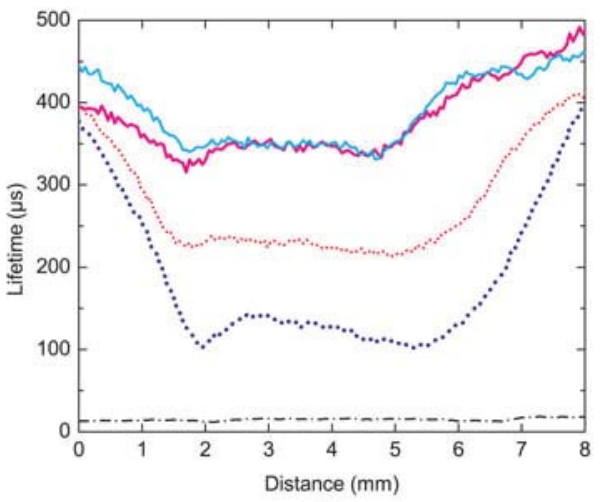

(c)

Fig. 5. Linescans marked in Fig. 3. One scan is performed on each position: D, F, and C. (a) Position D-Linescan 1. (b) Position F-Linescan 2. (c) Position C-Linescan 3.

Thus, recombination activity between structural defects and grains does not strongly deviate, resulting in low PL contrasts. During gettering, mobile impurities are removed, but still many of them preferably remain inside structural defects (or are internally gettered there), which results in highly recombination active dark PL regions. Thus, these structural defects are visible much more pronounced after gettering than in the as-grown state.

It is interesting to note for positions $\mathrm{F}$ and $\mathrm{C}$ that the difference between reference and industry diffusion vanishes after $\mathrm{SiN}_{x}+$ emitter firing, whereas for position $\mathrm{D}$, both diffusions still differ, with reference $+\mathrm{H}$ resulting in higher lifetimes. As 

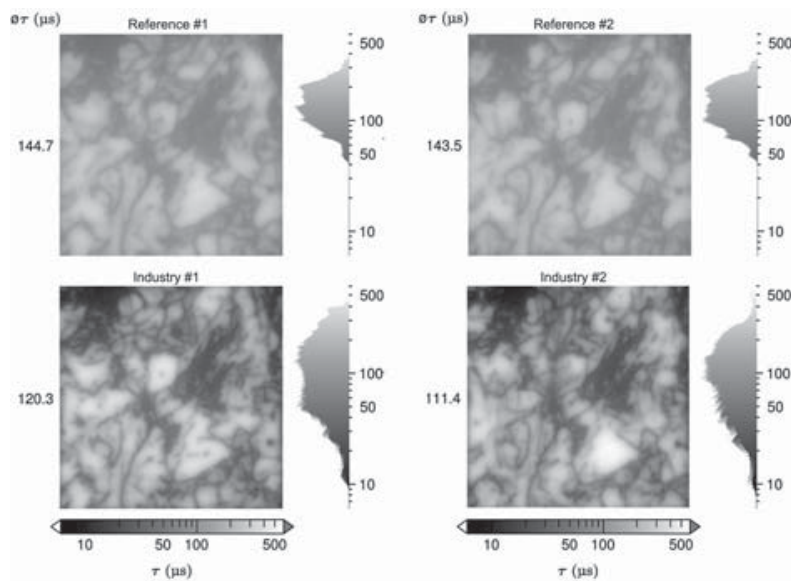

Fig. 6. Lifetime-calibrated PL maps of position D in central ingot height after two kinds of $\mathrm{POCl}_{3}$ gettering: Reference (top) and industry (bottom). Two neighboring samples (\#1 and \#2) per diffusion are shown.
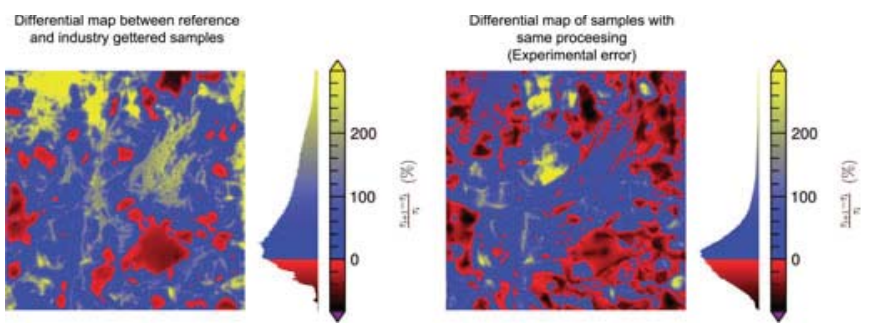

Fig. 7. Normalized differential maps of position D sister samples in Fig. 6. (Left) "Reference"-.'Industry." (Right) "Industry \# 1" - "Industry \#2."

already mentioned, positions $\mathrm{F}$ and $\mathrm{C}$ are gettered more effectively than position D due to their specific grain structure. After the additional gettering and/or hydrogenation step while firing $\mathrm{SiN}_{x}: \mathrm{H}$, highest lifetimes are reached for both diffusion types and this might saturate at a certain maximum level. Therefore, both diffusions in combination with the $\mathrm{SiN}_{x}: \mathrm{H}$ deposition and firing step seem to be capable of achieving this maximum lifetime on positions $\mathrm{C}$ and $\mathrm{F}$. On the contrary, at position $\mathrm{D}$, still nongettered and/or nonpassivated impurities, lowering the local lifetime, might remain in defect clusters especially for the shorter industry diffusion. Hence, the difference between both diffusions is maintained after $\mathrm{SiN}_{x}+$ emitter firing.

\section{Gettering Analysis on Position D Using Differential Maps}

1) Comparison of Two $\mathrm{POCl}_{3}$ Diffusions: Fig. 6 shows lifetime-calibrated PL maps of D samples in central ingot height. It is clearly seen that reference diffusion gettered samples exhibit higher lifetimes than industry gettered ones. To examine in more detail which regions actually improve, a differential map is calculated between reference $(i+1)$ and industry $(i)$ diffusion gettered sister samples [see Fig. 7(left)]. The shown deviation is normalized to the lower lifetime (industry diffused sample) according to the following equation:

$$
\tau_{\text {differential }}=\frac{\tau_{\mathrm{i}+1}-\tau_{\mathrm{i}}}{\tau_{\mathrm{i}}}
$$

The map on the right-hand side is the normalized differential between two samples that are processed in the same diffusion in order to see process-related deviations between sister samples. The full width at half maximum of its histogram is around $87 \%$. Assuming a normal distribution, this value corresponds to a standard deviation of $\pm 37 \%$. Improvements in the left-hand side map exceeding this deviation are relevant. Therefore, the mean improvement by the reference diffusion is $66(37) \%$. In particular, red regions are negative values, which would describe a degradation due to reference diffusion compared with industry diffusion. These values mainly constitute within the experimental error of $\pm 37 \%$ and, hence, may not necessarily reflect actual degradations. Nevertheless, it is interesting to note that most of these red regions belong to higher quality regions in the PL images. This implies that the shorter industry diffusion is more effective in regions of higher material quality. Yellow regions, describing a difference of $\approx 200 \%$ and higher, are correlated with dark black regions that do not improve significantly after industry diffusion compared with the as-grown state (see Figs. 5(a) and 6). These regions of low material quality might be accumulations of dislocations and GBs decorated with impurities lowering the bulk lifetime [25]. Since the initial as-grown lifetime within these regions is very low, it is not surprising that there is stronger percentage improvement than within regions of better initial material quality. Here, we focus on two kinds of diffusions which obviously lead to different final lifetimes within these regions starting from the same initial low lifetime. As a similar structure is visible after both reference and industry diffusion, significant structural changes due to the longer diffusion duration can be excluded. Reference gettered samples exhibit a much lower PL contrast than industry gettered ones. This indicates a more efficient improvement of low-quality regions, which consequently appear less pronounced. It is supposed that a higher impurity decoration of GBs and defect clusters is still present after the shorter industry process.

2) Effect of $\operatorname{SiN}_{x}: H+$ Emitter Firing: In this section, the effect of $\operatorname{SiN}_{x}: \mathrm{H}$ deposition and subsequently firing this layer with emitter underneath is discussed. It should be mentioned that from this experiment, it is not distinguishable if the analyzed effect originates from hydrogen in-diffusion, from additional $\mathrm{POCl}_{3}$ gettering or from a combination of both. The differential map between a reference gettered sample and its sister sample after the additional $\mathrm{SiN}_{x}: \mathrm{H}$ step is calculated. Samples are also D samples out of central ingot height and, therefore, are comparable with the already shown PL maps in Fig. 6. As can be seen from Fig. 8, there is an enhancement of bulk lifetime within the whole wafer. Again the experimental error is depicted on the right-hand side map. The standard deviation is only $12 \%$, which is significantly lower than the above shown error of samples after diffusion. According to the differential map in Fig. 8(left), it is remarkable that the effect of the $\operatorname{SiN}_{x}: H$ firing step predominantly improves regions that have not already been improved by reference gettering compared with industry gettering. These regions are highlighted in yellow, representing values up to $200 \%$, and correspond to individual GBs mostly in the vicinity of or even surrounding already reference gettered regions. An additional mean lifetime improvement of 98(12)\% 


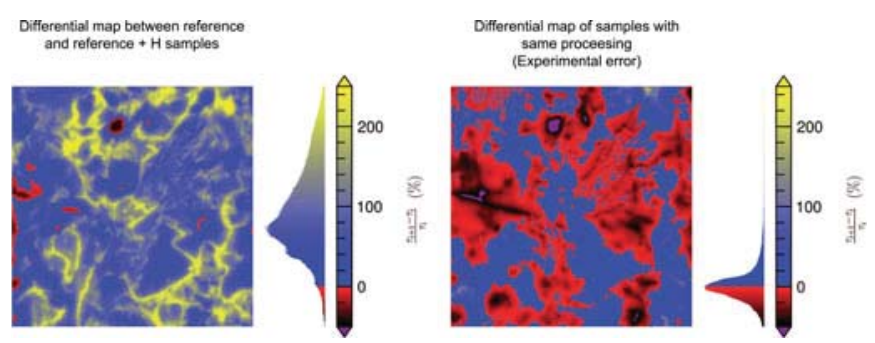

Fig. 8. Normalized differential map of reference gettered D samples with and without $\mathrm{SiN}_{x}: \mathrm{H}$ step. Sister samples of samples in Fig. 6 are depicted. (Left) "Reference + H"-"Reference." (Right) "Reference + H \#1" - "Reference + H \#2."

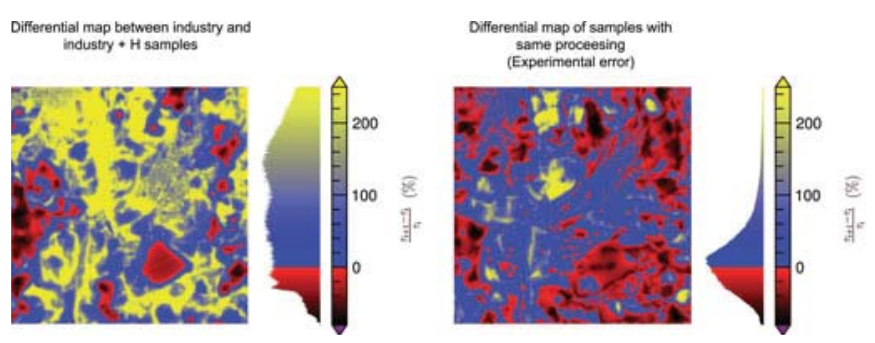

Fig. 9. Normalized differential map of industry gettered D samples with and without $\mathrm{SiN}_{x}: \mathrm{H}$ step. Sister samples of samples in Fig. 6 are depicted. (Left) "Industry + H"-"Industry." (Right) "Industry \#1" - "Industry \#2."

by $\mathrm{SiN}_{x}: \mathrm{H}$ is reached. It seems that both processing steps, emitter diffusion and $\mathrm{SiN}_{x}: \mathrm{H}$ deposition with firing, complement one another. Within particular regions, the reference diffusion might not have been able to remove all impurities. During firing, the remaining impurities might be partially gettered, and subsequently, dangling bonds are passivated by hydrogen or impurities themselves are passivated by hydrogen.

At first sight, the differential between industry gettered and industry $+\mathrm{H}$ gettered samples shown in Fig. 9 seems to exhibit a stronger relative lifetime enhancement. This might be expected since the previously industry gettered sample is of lower material quality compared with the reference diffusion. Thus, high relative lifetime changes can be more easily achieved starting from lower lifetimes compared with samples of better initial quality. The mean improvement of industry gettered material by $\mathrm{SiN}_{x}: \mathrm{H}$ with $137(37) \%$ is higher than the additional improvement of previously reference gettered material. In this case, the experimental error could not be determined from two sister samples since only one sample after industry $+\mathrm{H}$ is measured. Therefore, the standard deviation of $\pm 37 \%$ is assumed, which has already been determined for industry gettering. As already argued, a high amount of impurities may remain predominantly within defect clusters and GBs, which could not be gettered by the short industry diffusion before. Therefore, this amount of impurities might undergo another gettering process during $\mathrm{SiN}_{x}: \mathrm{H}$ firing and/or a passivation by hydrogen. It was shown in the previous Section III-B that positions C and F react to $\mathrm{POCl}_{3}$ diffusion differently than position D. They exhibit such strong lifetime improvements after industry $+\mathrm{H}$ gettering that the final lifetimes reach the level of reference $+\mathrm{H}$ gettered samples. These differences in responding to the same process schemes are attributed to the different defect structure for the different wafer positions. The corresponding structures are clearly seen in Fig. 3. In conclusion, the optimum process scheme for position $\mathrm{D}$ within central ingot height can be stated as reference diffusion followed by $\mathrm{SiN}_{x}: \mathrm{H}$ deposition and firing.

\section{Gettering Analysis in Different Ingot Heights-A Comparison With Solar Cell Efficiencies}

Fig. 10 shows mean lifetimes arithmetically averaged over the respective $5 \times 5 \mathrm{~cm}^{2}$ samples versus ingot height after each process scheme. In central ingot height, two samples per process are measured. Lifetimes of positions D, F, and C are depicted. The reference diffusion gettered samples are displayed as red crosses and group B samples $\left(+\mathrm{SiN}_{x}: \mathrm{H}\right)$ as red stars. Lifetimes after industry diffusion are marked as green minus signs and with an additional $\mathrm{SiN}_{x}: \mathrm{H}$ step as green plus signs. Black dots are the initial as-grown lifetimes with no processing except saw damage removal, surface cleaning, and passivation required for lifetime measurements. Highest as-grown lifetime in central ingot height is $41 \mu$ s for position $\mathrm{D}$ followed by position $\mathrm{F}$ with $30 \mu \mathrm{s}$. Position $\mathrm{C}$ exhibits the lowest as-grown lifetime of $28 \mu \mathrm{s}$. Note that a few data points are missing due to broken samples.

In the framework of the project SolarWinS (see the acknowledgement), an industrial project partner (Sunways AG) produced solar cells using a standard industrial cell process out of every tenth $15.6 \times 15.6 \mathrm{~cm}^{2}$ wafer along the ingot height. In Fig. 10, normalized efficiencies of these cells are included as blue squares and are linked to the right $y$-axis. Given efficiencies are normalized to the highest efficiency measured for the mc-Si material under investigation. As already stated in Section III-B, position $\mathrm{F}$ (central ingot height) exhibits such a strong lifetime increase that industry $+\mathrm{H}$ samples even slightly outreach the lifetime level of reference $+\mathrm{H}$ samples. This is also visible in Fig. 10. Again position $\mathrm{C}$ shows similar behavior in central ingot height with reference $+\mathrm{H}$ and industry $+\mathrm{H}$ reaching similar lifetimes.

As solar cells are commonly passivated by PECVD $\operatorname{SiN}_{x}: \mathrm{H}$ and fired, it should be focused on lifetime values after diffusion $+\mathrm{H}$ when comparing with cell efficiencies. It is interesting to note that edge positions $\mathrm{F}$ and $\mathrm{C}$ follow the same decrease in lifetime toward top ingot height as the solar cell efficiency. This is not observed for position D, probably because of the fact that position $\mathrm{D}$ is located closer to the ingot center, whereas positions $\mathrm{F}$ and $\mathrm{C}$ are closer to crucible walls. Therefore, it is indicated that $15.6 \times 15.6 \mathrm{~cm}^{2}$ solar cells in top ingot height are limited by the red zone which is the low material quality region originating from impurities diffusing out of crucible walls. It was shown by the detailed gettering analysis that the particular defect structure found in each sample determines the sample's behavior under varying PDG processes (compare, e.g., with similar results in [26] and [27]). The amount of extended defects especially limits PDG on position D. It should be emphasized here that comparing lifetimes with solar cell efficiencies is only possible by focusing on lifetime samples that 

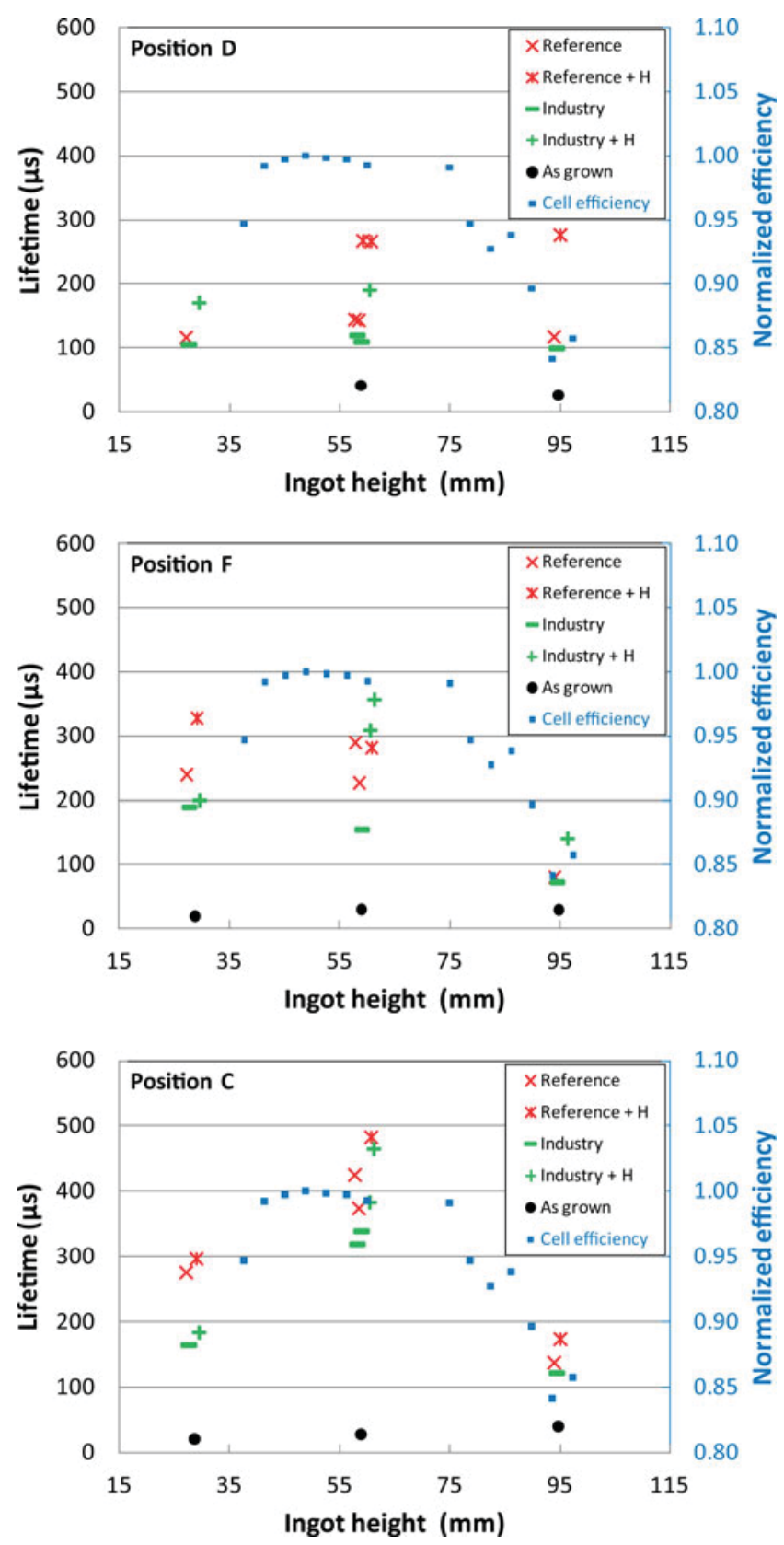

Fig. 10. Mean lifetimes versus ingot height at positions D, F, and C. Largearea solar cell efficiencies $(15.6 \mathrm{~cm} \times 15.6 \mathrm{~cm})$ are normalized to the highest efficiency reached for the mc-Si material under investigation. The physical ingot height, including the red zone, is given.

reflect the combination of gettering and hydrogenation, namely diffusion $+\mathrm{H}$ samples. Efficiency of large-area solar cells shows the same trend as the edge samples in contrast with the centrally positioned D sample. This behavior seems to be independent of the varying PDG processes. Each position, as well as each ingot height, exhibits a different optimum process that leads to the highest lifetime. This clearly shows the need of analyzing not only the difference in PDG efficacy but rather the combination of both PDG and hydrogenation. In the previous section, a fundamental difference between both processing steps is highlighted for the reference diffusion on position D (see Fig. 8).
The hydrogenation step herein predominantly improves GBs, while the optimized PDG alone (reference) is more efficient at extended defects. In summary, the achievable lifetime after the complete processing is not only dependent on the respective local defect structure but also on ingot height and position. The correlation in performance between lifetime edge samples and solar cells demonstrates that cell efficiencies are governed by the red zone. In addition, the material quality of edge samples is limited toward top ingot height independently of the applied type of PDG.

\section{DISCUSSION}

Comparing all three as-grown wafers in Fig. 3, it can be noticed that position $\mathrm{C}$ contains most twin boundaries running parallel for quite long distances (see enlarged section in Fig. 4). Also remarkable is a smaller number of grains compared with position $\mathrm{F}$ and especially to position $\mathrm{D}$, which contains the highest number of grains and smallest amount of GBs running parallel. According to Karzel et al. [28], it is suggested that these twin boundaries are $\sum 3 \mathrm{GBs}$ that exhibit a weaker recombination activity than for example GBs of higher $\sum$ value or dislocation clusters [29]. Chen et al. claimed that this type of GBs is only recombination active when contaminated, e.g., with $\mathrm{Fe}$ [30]. This is consistent with the presented results on position $\mathrm{C}$. Herein, most $\sum 3 \mathrm{GBs}$ are detected in the as-grown state, whereas their PL contrast vanishes after gettering. It can be argued that impurities are completely removed out of these grain boundaries. Because of the small tilt angle between neighboring grain orientations in case of $\sum 3 \mathrm{GBs}$, the crystal structure is less distorted than for higher $\sum$ values. Thus, it seems plausible that impurities are easily removed out of them. However, it is emphasized that the GB characterization via coincidence site lattice CSL ( $\sum$ value) is incomplete when classifying grain boundaries by their recombination activity. This is due to the fact that the $\sum$ value does not describe the boundary plane that is formed between the neighboring grains [31], [32]. Whether grain boundaries are recombination active or not is, therefore, highly discussed and not completely understood up to now.

The fact that the sample with most grains of smaller size exhibits the highest as-grown lifetime (position D) is not clear from first sight. It might be a consequence of an internal gettering effect which preferably occurs at grain boundaries during crystallization and cooling. Due to this effect, intragrain regions of position D might be cleaner and reach higher bulk lifetimes than intragrain regions of position $\mathrm{F}$, for example. At position $\mathrm{F}$, these regions are crossed by the aforementioned twin boundaries $\left(\sum 3\right)$ and appear as blurry dark regions of low material quality in the as-grown PL image. In addition to the fact that position D exhibits most structural defects serving as many internal gettering sites, the edge samples $\mathrm{C}$ and $\mathrm{F}$ are more strongly and homogeneously contaminated by in-diffusing impurities from the crucible walls. In comparison with that, position D exhibits intragrain regions of brighter PL contrasts and therefore higher lifetimes. After gettering, the situation is completely changed. The best gettering efficacy occurs for the $\mathrm{C}$ samples with the lowest as-grown lifetimes, but the highest number of $\sum 3 \mathrm{GBs}$. This would lead to the conclusion of a facilitated gettering 
process when there are more $\sum 3$ GBs present in the material. In addition, it can be stated here that the initial as-grown lifetime alone is not a good indicator for predicting the gettering efficacy of mc-Si, without taking the local defect structure into account.

According to Fig. 7, the longer lasting $\mathrm{POCl}_{3}$ diffusion process (reference) predominantly improves the already mentioned defect clusters highlighted in yellow. These PDG-limiting extended defects that are selected by the linescans in Fig. 5 have been exposed to a defect etch. A clear correlation between higher dislocation density and recombination activity can be drawn from these measurements (not shown here). A critical dislocation density of $10^{6} 1 / \mathrm{cm}^{2}$ is detected, which can be seen as the minimum dislocation density leading to a lowered lifetime compared with nondislocated surroundings. Lattice defects like dislocations and grain boundaries can enhance impurity diffusion since activation energy for migrating atoms can be lowered in these regions depending on the type of foreign atom [33], [34]. On the contrary, they might also serve as internal gettering sites with dangling bonds and vacancies that attract and facilitate precipitation of impurities [6], [35]. If impurities stay recombination active within these sites, the external $\mathrm{POCl}_{3}$ gettering process is constricted. In the case of a decelerated precipitation dissolution and impurity diffusion, atoms need more time and/or higher temperature to be released from lattice defects, particularly from defect clusters, and to be transported toward external gettering sites. Since both diffusions are performed at the same temperature, the longer lasting reference diffusion might be better suited. Additionally, there is a higher oxygen flow during drive-in. Oxygen is found to produce a large amount of $\mathrm{Si}$ self-interstitials due to amorphous $\mathrm{SiO}_{2}$ formation with a volume expansion of $\approx 130 \%$ [36], [37]. It is suggested that the supersaturation of these interstitials is responsible for gettering via the so-called kick-out mechanism [38]-[40]. Another possible mechanism behind $\mathrm{P}$ gettering is given by Syre et al. [8]. Here, vacancies are claimed to be responsible for gettering while they are formed during $\mathrm{P}$ in-diffusion in the near-surface region containing very high $\mathrm{P}$ concentration. Vacancy and oxygen form $\mathrm{VO}_{2}$ complexes that might act as precipitation centers for oxygen precipitates which, in turn, act as gettering sites for Fe. Consequently, it seems plausible that a longer drive-in time accompanied by a higher oxygen flow provides a more efficient gettering diffusion.

\section{SUMMARY}

Different gettering efficacy is observed for two $80-\Omega / \square$ $\mathrm{POCl}_{3}$ emitters on mc-Si carried out with different drive-in durations and gas flows. The extended diffusion combined with a higher oxygen flow results in higher bulk lifetimes.

A detailed gettering analysis of three positions (D, F, and C) out of a $15.6 \times 15.6 \mathrm{~cm}^{2}$ wafer leads to the conclusion that the particular defect structure of each position determines its behavior under varied $\mathrm{POCl}_{3}$ diffusions. This is most evident for central ingot height of position $\mathrm{C}$, which contains less defect clusters compared with the other positions and reaches, by far, the highest lifetimes after gettering.
A comparison with efficiencies of $15.6 \times 15.6 \mathrm{~cm}^{2}$ solar cells shows that lifetimes of samples originating from wafer edge regions $(\mathrm{C}$ and $\mathrm{F})$ follow the same decreasing trend toward the top ingot height. On the contrary, D samples are closer to the ingot center and do not reflect that decrease. It is suggested that cell efficiencies are limited by the red zone, in which edge wafers are located.

\section{ACKNOWLEDGMENT}

The authors would like to thank S. Joos and N. Brinkmann for their help concerning surface passivation issues. Technical support by L. Mahlstaedt is also gratefully acknowledged. The content is the responsibility of the authors.

\section{REFERENCES}

[1] A. Cuevas, "Lifetime studies of multicrystalline silicon," in Proc. NREL Workshop Crystalline Silicon PV, pp. 50-59, 1998.

[2] D. Macdonald, A. Cuevas, and F. Ferazza, "Response to phosphorus gettering of different regions of cast multicrystalline silicon ingots," Solid-State Electron., vol. 43, pp. 575-581, 1999.

[3] J. Tan, A. Cuevas, D. Macdonald, T. Trupke, R. Bardos, and K. Roth, "On the electronic improvement of multi-crystalline silicon via gettering and hydrogenation," Progr. Photovoltaic., Res. Appl., vol. 16, pp. 129-134, 2008.

[4] D. Macdonald, A. Cuevas, A. Kinomura, and Y. Nakano, "Phosphorus gettering in multicrystalline silicon studied by neutron activation analysis," in Proc. 29th IEEE Photovoltaic Spec. Conf., 2002, pp.285-288.

[5] A. Istratov, T. Buonassisi, R. McDonald, A. Smith, R. Schindler, J. Rand, J. Kalejs, and E. Weber, "Metal content of multicrystalline silicon for solar cells and its impact on minority carrier diffusion length," J. Appl. Phys., vol. 94, no. 10, pp. 6552-6559, 2003.

[6] F. Salman, J. Arnold, P. Zhang, G. Chai, F. Stevie, and L. Chow, "Redistribution of implanted species in polycrystalline silicon films on silicon substrate," Defect Diffus. Forum, vol. 264, pp. 7-12, 2007.

[7] A. Bentzen, J. Christensen, B. Svensson, and A. Holt, "Understanding phosphorus emitter diffusion in silicon solar cell processing," in Proc. 21th Eur. Photovoltaic Sol. Energy Conf. Exhib., 2006, pp. 1388-1391.

[8] M. Syre, S. Karazhanov, B. Olaisen, A. Holt, and B. Svensson, "Evaluation of possible mechanisms behind P gettering of iron," J. Appl. Phys., vol. 110, no. 2, pp. 024912-1-024912-5, 2011.

[9] D. Kumar, S. Saravanan, and P. Suratkar, "Effect of oxygen ambient during phosphorous diffusion on silicon solar cell," J. Renewable Sustainable Energy, vol. 4, no. 3, pp. 033105-1-033105-8, 2012.

[10] D. Macdonald, J. Tan, and T. Trupke, "Imaging interstitial iron concentrations in boron-doped crystalline silicon using photoluminescence," J. Appl. Phys., vol. 103, no. 7, pp. 073710-1-073710-7, 2008.

[11] M. Seibt, D. Abdelbarey, V. Kveder, C. Rudolf, P. Saring, L. Stolze, and $\mathrm{O}$. Voß, "Interaction of metal impurities with extended defects in crystalline silicon and its implications for gettering techniques used in photovoltaics," Mater. Sci. Eng. B, vol. 160, pp. 264-268, 2009.

[12] R. Sinton and A. Cuevas, "Contactless determination of current-voltage characteristics and minority-carrier lifetimes in semiconductors from quasi-steady-state photoconductance data," Appl. Phys. Lett., vol. 69, no. 17, pp. 2510-2512, 1996.

[13] T. Trupke, R. Bardos, M. Schubert, and W. Warta, "Photoluminescence imaging of silicon wafers," Appl. Phys. Lett., vol. 89, no. 4, pp. $044107-$ $1-044107-3,2006$.

[14] T. Buonassisi, D. Pickett, and R. Sweeney, "Reducing the concentration of interstitial iron in crystalline silicon solar cells via low $\left(<500{ }^{\circ} \mathrm{C}\right)$ temperature annealing," in Proc. NREL Workshop Cryst. Silicon PV, pp. 217-220, 2007.

[15] M. Rinio, A. Yodyunyong, S. Keipert-Colberg, Y. Botchak Mouafi, D. Borchert, and A. Montesdeoca-Santana, "Improvement of multicrystalline silicon solar cells by a low temperature anneal after emitter diffusion," Progr. Photovoltaic, Res. Appl., vol. 19, no. 2, pp. 165-169, 2010.

[16] J. Schön, H. Habenicht, M. Schubert, and W. Warta, "Understanding the distribution of iron in multicrystalline silicon after emitter formation: Theoretical model and experiments," J. Appl. Phys., vol. 109, no. 6, pp. 063717-1-063717-8, 2011. 
[17] M. Seibt and V. Kveder, "Gettering processes and the role of extended defects," in Advanced Silicon Materials for Photovoltaic Applications, S. Pizzini, Ed. Chichester, U.K.: Wiley, 2012, ch. 4, pp.126-188.

[18] J. Kang and D. Schroder, "Gettering in silicon," J. Appl. Phys., vol. 65, no. 8, pp. 2974-2985, 1989.

[19] D. Gilles, W. Schröter, and W. Bergholz, "Impact of the electronic structure on the solubility and diffusion of 3D transition elements in silicon," Phys. Rev. B, vol. 41, no. 9, pp. 5770-5782, 1990.

[20] S. Myers, M. Seibt, and W. Schröter, "Mechanisms of transition-metal gettering in silicon," J. Appl. Phys., vol. 88, no. 7, pp. 3795-3819, 2000.

[21] V. Kveder, M. Seibt, A. Sattler, and E. Spiecker, "Mechanisms and computer modelling of transition element gettering in silicon," Sol. Energy Mater, vol. 72, pp. 299-313, 2002

[22] S. Dauwe, J. Schmidt, and R. Hezel, "Very low surface recombination velocities on p-and n-type silicon wafers passivated with hydrogenated amorphous silicon films," in Proc. 29th IEEE Photovoltaic Spec. Conf., 2002, pp.1246-1249.

[23] D. Kohler, A. Zuschlag, and G. Hahn, "On the origin and formation of large defect clusters in multicrystalline silicon solar cells," Sol. Energy Mater. Sol. Cells, vol. 120, pp. 275-281, Jan. 2014.

[24] D. Fenning, A. Zuschlag, A. Frey, M. Bertoni, B. Lai, G. Hahn, and T. Buonassisi, "Identification of lifetime-limiting defects after hightemperature phosphorus diffusion in silicon solar cell materials." IEEE J. Photovolt., vol. 4, no. 3, pp. 866-873, 2014.

[25] H. Sugimoto, M. Inoue, M. Tajima, A. Ogura, and Y. Ohshita, "Analysis of intra-grain defects in multicrystalline silicon wafers by photoluminescence mapping and spectroscopy," Jpn. J. Appl. Phys., vol. 45, no. 25, pp. L641L643, Jun. 2006.

[26] P. Geiger, G. Kragler, G. Hahn, and P. Fath, "Spatially resolved investigations of lifetime enhancement in vertically grown, multicrystalline silicon ribbons," Sol. Energy Mater. Sol. Cells, vol. 85, no. 4, pp. 559-572, Feb. 2005

[27] A. Bentzen, A. Holt, R. Kopecek, G. Stokkan, J. S. Christensen, and B. G. Svensson, "Gettering of transition metal impurities during phosphorus emitter diffusion in multicrystalline silicon solar cell processing," $J$. Appl. Phys., vol. 99, no. 9, pp. 093509-1-093509-6, 2006.

[28] P. Karzel, M. Ackermann, L. Groner, C. Reimann, M. Zschorsch, S. Meyer, F. Kiessling, S. Riepe, and G. Hahn, "Dependence of phosphorus gettering and hydrogen passivation efficacy on grain boundary type in multicrystalline silicon," J. Appl. Phys., vol. 114, no. 24, pp. 244902-1-244902-11, 2013.

[29] H. Sio, T. Trupke, S. Phang, and D. Macdonald, "Electrical properties of different types of grain boundaries in multicrystalline silicon by photoluminescence imaging," in Proc. 27th Eur. Photovoltaic Sol. Energy Conf. Exhib., 2012, pp. 714-718.

[30] B. Chen, J. Chen, T. Sekiguchi, M. Saito, and K. Kimoto, "Structural characterization and iron detection at $\Sigma 3$ grain boundaries in multicrystalline silicon," J. Appl. Phys., vol. 105, no. 11, pp. 113502-1-113502-5, 2009.

[31] V. Randle, "A methodology for grain boundary plane assessment by singlesection trace analysis," Scripta Materialia, vol. 44, no. 12, pp. 2789-2794, Jun. 2001

[32] M. Tschopp and D. McDowell, "Structural unit and faceting description of $\Sigma 3$ asymmetric tilt grain boundaries," J. Mater. Sci., vol. 42, no. 18, pp. 7806-7811, May. 2007.

[33] H. Queisser, K. Hubner, and W. Shockley, "Diffusion along small-angle grain boundaries in silicon," Phys. Rev., vol. 123, no. 4, pp. 1245-1254, 1961.

[34] T. Kamins, "Diffusion of impurities in polycrystalline silicon," J. Appl. Phys., vol. 43, no. 1, pp. 83-91, 1972.

[35] J. Chen, T. Sekiguchi, D. Yang, F. Yin, K. Kido, and S. Tsurekawa, "Electron-beam-induced current study of grain boundaries in multicrystalline silicon," J. Appl. Phys., vol. 96, no. 10, pp. 5490-5495, 2004.

[36] S. Mahajan and K. SreeHarsha, Principles of Growth and Processing of Semiconductors. New York, NY, USA: WCB/McGraw-Hill, 1999.

[37] X. Zhang, Electrochemistry of Silicon and Its Oxide. New York, NY, USA: Kluwer, 2001

[38] A. Ourmazd and W. Schröter "Phosphorus gettering and intrinsic gettering of nickel in silicon," Appl. Phys. Lett., vol. 45, no. 7, pp. 781-783, 1984.

[39] W. Schröter, A. Döller, A. Zozime, V. Kveder, M. Seibt, and E. Spiecker, "Phosphorus diffusion gettering of metallic impurities in silicon: mechanisms beyond segregation," Solid State Phenomena, vols. 95/96, pp. $527-538,2004$.

[40] P. Fahey, P. Griffin, and J. Plummer, "Point defects and dopant diffusion in silicon," J. ICRU, vol. 11, no. 2, p. 11, Dec. 2011.

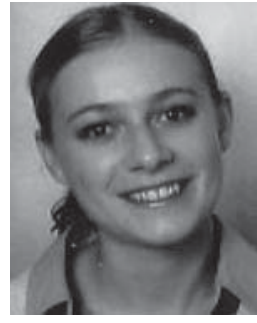

gettering.
Sarah Gindner received the Diploma degree in physics from the University of Konstanz, Konstanz, Germany, in 2008 after preparing her thesis externally at the group of Prof. Dr. Wuttig with the I. Physikalisches Insitut, RWTH Aachen, Aachen, Germany. She is currently working toward the Ph.D. degree in the field of photovoltaics under Dr. G. Hahn with the University of Konstanz.

Her major research interests include material quality limiting defects in multicrystalline silicon and material changes caused by phosphorus diffusion

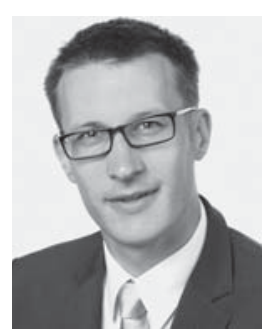

Philipp Karzel received the Diploma degree in physics from the University of Konstanz, Konstanz, Germany, in 2008, where he is currently working toward the Ph.D. degree in the photovoltaics group of Prof. G. Hahn.

His research interests include defect engineering in multicrystalline silicon, specifically focussing on the interaction of hydrogen with interstitial iron and grain boundaries.

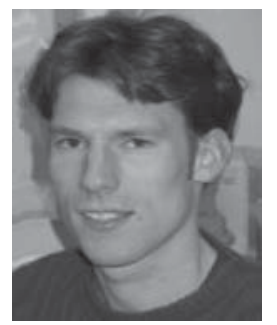

Bernhard Herzog received the Diploma degree in physics with the University of Erlangen, Germany, in 2003. He is currently working toward the Ph.D. degree in the photovoltaics group of Prof. G. Hahn with the University of Konstanz, Konstanz, Germany.

His research interests include defect engineering and material quality investigations in standard and compensated multicrystalline silicon.

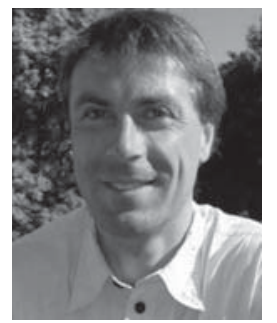

Giso Hahn was born in Frankfurt am Main, Germany, in 1969. He received the Diploma degree in physics from the University of Stuttgart, Stuttgart, Germany, in 1995 and the Ph.D. degree in physics from the University of Konstanz, Konstanz, Germany, in 1999.

Since 2009, he has been an Apl. Professor with the Department of Physics, University of Konstanz. His research interests include crystalline silicon materials and solar cell process development, including characterization of promising low-cost materials for photovoltaic applications and the development of adapted solar cell processes for these and other materials.

Dr. Hahn is a Member of the scientific committees of various conference series (e.g., IEEE Photovoltaic Specialists Conference, European Photovoltaic Solar Energy Conference, International Conference on Silicon Photovoltaics) and workshops. 